\title{
Nonlinear models for transverse forced vibration of axially moving viscoelastic beams
}

\author{
Hu Ding ${ }^{\mathrm{a}, *}$ and Li-Qun Chen ${ }^{\mathrm{a}, \mathrm{b}}$ \\ a Shanghai Institute of Applied Mathematics and Mechanics, Shanghai University, Shanghai, 200077, China \\ ${ }^{\mathrm{b}}$ Department of Mechanics, Shanghai University, Shanghai, 200444, China
}

Received 2 February 2010

Revised 17 May 2010

\begin{abstract}
Nonlinear models of transverse vibration of axially moving viscoelastic beams subjected external transverse loads via steady-state periodical response are numerically investigated. An integro-partial-differential equation and a partial-differential equation of transverse motion can be derived respectively from a model of the coupled planar vibration for an axially moving beam. The finite difference scheme is developed to calculate steady-state response for the model of coupled planar and the two models of transverse motion under the simple support boundary. Numerical results indicate that the amplitude of the steady-state response for the model of coupled vibration and two models of transverse vibration predict qualitatively the same tendencies with the changing parameters and the integro-partial-differential equation gives results more closely to the coupled planar vibration.
\end{abstract}

Keywords: Vibration, nonlinearity, axially moving beam, viscoelasticity, finite difference

\section{Introduction}

Axially moving beams are extensively investigated because they can model many engineering devices such as magnetic tapes, plastic films, power transmission belts, textile fibers and paper sheets. Under certain conditions, an axially moving beam may undergo transverse and longitudinal motions that are usually coupled if the geometrical nonlinearity has to be considered. Thurman and Mote first developed the governing equations of planar motion of axially moving beams [1]. Under certain conditions, nonlinear models for transverse motion can be obtained to govern the transverse motion via the transverse motion are decoupled from the longitudinal motion. Under the quasistatic stretch assumption, Wickert developed a nonlinear model for transverse motion of axially moving beams [2]. The approach is also referred as Kirchhoff's approach [3]. The model was respectively applied to analyze chaotic response [4], parametrically excited [5-8], parametric instability [9], complex dynamic [10], free vibration [11,12], and forced vibration [13] of transverse vibration of axially moving beams. Another nonlinear model for transverse motion can be derived from the coupled model by setting all longitudinal term zero and omitting higher order nonlinear terms. This model has been used to investigate steady-state response of Parametric resonance stability [6, 7,14-16], internal resonance [17], nonlinear dynamic [18], free vibration [11], forced vibration, and forced vibration internal damping [19] of axially moving beams. These two transverse vibration models for axially moving beams are respectively a nonlinear integro-partial-differential equation and a nonlinear partial-differential equation.

Very limited attentions have been paid to the difference between the integro-differential equation and the partialdifferential equation. Approximate analytical results based on the two models were compared for steady-state

\footnotetext{
*Corresponding author. E-mail: dinghu3@ shu.edu.cn.
} 
response of parametric vibration $[6,7,15]$ and free vibration $[11,12,20]$ of axially moving beams. It is found that the predictions made by the two models are qualitatively the same, but quantitatively different. However, so far it has not been clear which model yields better outcomes.

To examine the validity of the two nonlinear models and to determine the superiority in the sense of approximating the coupled governing equation of planar vibration, the transverse responses calculated numerically from two models were respectively compared with the transverse component calculated from the coupled equation for free vibration of axially moving strings [21] and beams under both the simple support boundary and the fixed boundary conditions [20], and also with the steady-state responses, which were calculated from the three nonlinear models of axially moving viscoelastic beams subjected external transverse loads under the fixed boundary conditions [22], and the comparison results indicated that the integro-differential equation can provide closer results to the coupled equation. The goal of the present investigation is to examine the validity of the two nonlinear models of axially moving viscoelastic beams subjected external transverse loads and to determine the superiority under the simple support boundary.

The present paper is organized as follows. Section 1 establishes the integro-partial-differential model and the partial-differential equation model for the transverse motion from the coupled model of an axially moving beam. Section 2 presents the finite difference scheme to solve the governing equations numerically. Section 3 presents numerical results of the steady-state periodical response calculated from these models presented in Section 1, and compares the coupled equations of planar motion with two governing equations of transverse motion via the steady-state periodical response. Section 4 ends the paper with the concluding remarks.

\section{Mathematical formulations}

Consider a uniform axially moving beam of density $\rho$, initial tension $P_{0}$, cross-sectional area $A$, and moment of inertial $I$. The beam travels at the uniform constant transport speed $c$ between two boundaries separated by distance $l$. The distance from the left boundary is measured by fixed axial coordinate $x$. Assume that the deformation of the beam is confined to the vertical plane. The beam is subjected to an external transverse excitation $F(x, t)$ only, where $t$ is the time. The in-plane motion of the beam is specified by the transverse displacement $v(x, t)$ and the longitudinal displacement $u(x, t)$, the viscoelastic material of the beam obeys the Kelvin model [7], for a slender beam (for example, with $I /\left(A l^{2}\right)<0.001$ ), the Newton second law of motion yields

$$
\begin{gathered}
\rho A\left(\frac{\partial^{2} u}{\partial t^{2}}+2 c \frac{\partial^{2} u}{\partial x \partial t}+c^{2} \frac{\partial^{2} u}{\partial x^{2}}\right)-\frac{\partial}{\partial x} \frac{\left(P_{0}+A E \varepsilon+A \eta \frac{\partial \varepsilon}{\partial t}+A \eta c \frac{\partial \varepsilon}{\partial x}\right)\left(1+\frac{\partial u}{\partial x}\right)}{\sqrt{\left(1+\frac{\partial u}{\partial x}\right)^{2}+\left(\frac{\partial v}{\partial x}\right)^{2}}}=0 \\
\rho A\left(\frac{\partial^{2} v}{\partial t^{2}}+2 c \frac{\partial^{2} v}{\partial x \partial t}+c^{2} \frac{\partial^{2} v}{\partial x^{2}}\right)-\frac{\partial}{\partial x} \frac{\left(P_{0}+A E \varepsilon+A \eta \frac{\partial \varepsilon}{\partial t}+A \eta c \frac{\partial \varepsilon}{\partial x}\right) \frac{\partial v}{\partial x}}{\sqrt{\left(1+\frac{\partial u}{\partial x}\right)^{2}+\left(\frac{\partial v}{\partial x}\right)^{2}}}+ \\
E I \frac{\partial^{4} v}{\partial x^{4}}+\eta I\left(\frac{\partial^{5} v}{\partial x^{4} t}+c \frac{\partial^{5} v}{\partial x^{5}}\right)=F(x, t)
\end{gathered}
$$

where a comma preceding $x$ or $t$ denotes partial differentiation with respect to $x$ or $t, E$ is the Young's modulus, $\eta$ is the dynamic viscosity, and the disturbed strain $\varepsilon(x, t)$ of the beam is given by the nonlinear geometric relation

$$
\varepsilon=\sqrt{\left(1+\frac{\partial u}{\partial x}\right)^{2}+\left(\frac{\partial v}{\partial x}\right)^{2}}-1
$$

In the following, a special form of external transverse excitation is considered. It is assumed that the excitation is spatially uniform and temporally harmonic. That is

$$
F(x, t)=b \cos (\omega t)
$$

where $b$ and $\omega$ are respectively the amplitude and the frequency of the external excitation.

To cast Eqs (1), (2), and (3) dimensionless, introduce the transformation of the time and the space coordinates 


$$
v \leftrightarrow \frac{v}{l}, u \leftrightarrow \frac{u}{l}, x \leftrightarrow \frac{x}{l}, b \leftrightarrow \frac{b}{l}, t \leftrightarrow t \sqrt{\frac{P_{0}}{\rho A l^{2}}}, \omega \leftrightarrow \omega \sqrt{\frac{\rho A L^{2}}{P_{0}}}
$$

and define new parameters

$$
\gamma=c \sqrt{\frac{\rho A}{P_{0}}}, k_{f}=\sqrt{\frac{E I}{P_{0} l^{2}}}, \alpha=\frac{I \eta}{L^{3} \sqrt{\rho A P_{0}}}, k_{1}=\sqrt{\frac{E A}{P_{0}}}, k_{2}=\frac{\eta \sqrt{A}}{l \sqrt{\rho P_{0}}}
$$

where $k_{f}^{2}$ accounts for the bending stiffness of the beam, dimensionless parameter $\alpha$ denotes the dynamic viscosity, dimensionless parameter $k_{1}$ represents the effect of nonlinearity, dimensionless parameter $k_{2}$ represents the effects of nonlinearity associated with the dynamic viscosity, $\gamma$ and $f(x, t)$ are the dimensionless axial speed and transverse load respectively. Under transformation (2), Eq. (1) can be expressed in the new parameters defined by Eq. (5) as

$$
\begin{gathered}
\frac{\partial^{2} u}{\partial t^{2}}+2 \gamma \frac{\partial^{2} u}{\partial x \partial t}+\gamma^{2} \frac{\partial^{2} u}{\partial x^{2}}=\frac{\partial}{\partial x} \frac{\left(1+k_{1}^{2} \varepsilon+k_{2} \frac{\partial \varepsilon}{\partial t}+\gamma k_{2} \frac{\partial \varepsilon}{\partial x}\right)\left(1+\frac{\partial u}{\partial x}\right)}{\varepsilon+1} \\
\frac{\partial^{2} v}{\partial t^{2}}+2 \gamma \frac{\partial^{2} v}{\partial x \partial t}+\gamma^{2} \frac{\partial^{2} v}{\partial x^{2}}+k_{f}^{2} \frac{\partial^{4} v}{\partial x^{4}}+\alpha \frac{\partial^{5} v}{\partial x^{4} \partial t}+\alpha \gamma \frac{\partial^{5} v}{\partial x^{5}}= \\
\frac{\partial}{\partial x} \frac{\left(1+k_{1}^{2} \varepsilon+k_{2} \frac{\partial \varepsilon}{\partial t}+\gamma k_{2} \frac{\partial \varepsilon}{\partial x}\right) \frac{\partial v}{\partial x}}{\varepsilon+1}+b \cos (\omega t)
\end{gathered}
$$

In small but finite stretching problems in literature of nonlinear oscillations, the coupling between transverse and longitudinal vibration is assumed negligible and only a few lower order nonlinear terms need to be retained. Inserting $u=0$ into Eq. (6) and omitting higher order nonlinear terms yield a governing equation of transverse vibration under an external load in the dimensionless form [22]

$$
\begin{aligned}
& \frac{\partial^{2} v}{\partial t^{2}}+2 \gamma \frac{\partial^{2} v}{\partial x \partial t}+\gamma^{2} \frac{\partial^{2} v}{\partial x^{2}}+k_{f}^{2} \frac{\partial^{4} v}{\partial x^{4}}+\alpha \frac{\partial^{5} v}{\partial x^{4} \partial t}+\alpha \gamma \frac{\partial^{5} v}{\partial x^{5}}= \\
& \frac{3}{2} k_{1}^{2} \frac{\partial v^{2}}{\partial x} \frac{\partial^{2} v}{\partial x^{2}}+k_{2} \alpha \frac{\partial v}{\partial x}\left(2 \frac{\partial^{2} v}{\partial x^{2}} \frac{\partial^{2} v}{\partial x \partial t}+\frac{\partial v}{\partial x} \frac{\partial^{3} v}{\partial x^{2} \partial t}+2 \gamma \frac{\partial^{2} v^{2}}{\partial x^{2}}+\gamma \frac{\partial v}{\partial x} \frac{\partial^{3} v}{\partial x^{3}}\right)+b \cos (\omega t)
\end{aligned}
$$

On the other hand, the quasi-static stretch assumption means that one can use the averaged value of the disturbed tension to replace the exact value. In this case, Eq. (7) becomes

$$
\begin{aligned}
& \frac{\partial^{2} v}{\partial t^{2}}+2 \gamma \frac{\partial^{2} v}{\partial x \partial t}+\gamma^{2} \frac{\partial^{2} v}{\partial x^{2}}+k_{f}^{2} \frac{\partial^{4} v}{\partial x^{4}}+\alpha \frac{\partial^{5} v}{\partial x^{4} \partial t}+\alpha \gamma \frac{\partial^{5} v}{\partial x^{5}}= \\
& {\left[1+\int_{0}^{l}\left(\frac{1}{2} k_{1}^{2} \frac{\partial v^{2}}{\partial x}+k_{2} \frac{\partial v}{\partial x} \frac{\partial^{2} v}{\partial x \partial t}+k_{2} \gamma \frac{\partial v}{\partial x} \frac{\partial^{2} v}{\partial x^{2}}\right) d x\right] \frac{\partial^{2} v}{\partial x^{2}}+b \cos (\omega t)}
\end{aligned}
$$

Equations (7) and (8) are two nonlinear models of transverse vibrations.

In the present investigation, only the simply supported at both ends boundary conditions are considered, the boundary conditions of the dimensionless form are [20]

$$
\begin{aligned}
& u(0, t)=u(l, t)=0 \\
& v(0, t)=v(l, t)=0,\left.\frac{\partial^{2} v}{\partial x^{2}}\right|_{x=0}=\left.\frac{\partial^{2} v}{\partial x^{2}}\right|_{x=l}=0
\end{aligned}
$$

\section{Numerical approach}

The finite difference method will be employed to solve numerically Eq. (6) with Eq. (2), and Eqs (7) and (8). Introduce the $L \times T$ equally spaced mesh grid with space step $h$ and time step $\tau$

$$
x_{j}=j h \quad(j=0,1,2, \ldots L, h=l / L), t_{n}=n \tau \quad(n=0,1,2, \ldots . T) .
$$


Denote the function values $u(x, t)$ and $v(x, t)$ at $\left(x_{j}, t_{n}\right)$ as $u_{j}^{n}$ and $v_{j}^{n}$. Applying the centered difference approximations to the time, space and mixed partial derivatives leads to [20]

$$
\begin{aligned}
\frac{\partial u}{\partial x} & =\frac{u_{j+1}^{n}-u_{j-1}^{n}}{2 h}, \frac{\partial^{2} u}{\partial t^{2}}=\frac{u_{j}^{n+1}-2 u_{j}^{n}+u_{j}^{n-1}}{\tau^{2}} \\
\frac{\partial^{2} u}{\partial x^{2}} & =\frac{u_{j+1}^{n}-2 u_{j}^{n}+u_{j-1}^{n}}{h^{2}}, \frac{\partial^{2} u}{\partial x \partial t}=\frac{u_{j+1}^{n+1}-u_{j+1}^{n-1}-u_{j-1}^{n+1}+u_{j-1}^{n-1}}{4 h \tau},
\end{aligned}
$$

and

$$
\begin{aligned}
\frac{\partial v}{\partial x} & =\frac{v_{j+1}^{n}-v_{j-1}^{n}}{2 h}, \frac{\partial^{2} v}{\partial t^{2}}=\frac{v_{j}^{n+1}-2 v_{j}^{n}+v_{j}^{n-1}}{\tau^{2}}, \\
\frac{\partial^{2} v}{\partial x^{2}} & =\frac{v_{j+1}^{n}-2 v_{j}^{n}+v_{j-1}^{n}}{h^{2}}, \frac{\partial^{2} v}{\partial x \partial t}=\frac{v_{j+1}^{n+1}-v_{j+1}^{n-1}-v_{j-1}^{n+1}+v_{j-1}^{n-1}}{4 h \tau}, \\
\frac{\partial^{3} v}{\partial x^{3}} & =\frac{-v_{j-2}^{n}+2 v_{j-1}^{n}-2 v_{j+1}^{n}+v_{j+2}^{n}, \frac{\partial^{4} v}{\partial x^{4}}=\frac{v_{j-2}^{n}-4 v_{j-1}^{n}+6 v_{j}^{n}-4 v_{j+1}^{n}+v_{j+2}^{n}}{h^{4}},}{\frac{\partial^{5} v}{\partial x^{4} \partial t}}=\frac{v_{j+2}^{n+1}-4 v_{j+1}^{n+1}+6 v_{j}^{n+1}-4 v_{j-1}^{n+1}+v_{j-2}^{n+1}-v_{j+2}^{n-1}+4 v_{j+1}^{n-1}-6 v_{j}^{n-1}+4 v_{j-1}^{n-1}-v_{j-2}^{n-1}}{2 \tau h^{4}}, \\
\frac{\partial^{5} v}{\partial x^{5}} & =\frac{-v_{j-3}^{n}+4 v_{j-2}^{n}-5 v_{j-1}^{n}+5 v_{j+1}^{n}-4 v_{j+2}^{n}+v_{j+3}^{n}}{2 h^{5}},
\end{aligned}
$$

Substitution of Eqs (12) and (13) into Eq. (6) leads to a set of algebraic equations with respect to $u_{j}^{n}$ and $v_{j}^{n}$ that can be solved as under the boundary conditions Eqs (9) and (10) for prescribed parameters and initial conditions. Then the resulting grid values $u_{j}^{n}$ and $v_{j}^{n}$ are used in the finite difference schemes as an approximation to the continuous solutions $u(x, t)$ and $v(x, t)$ to Eq. (6). Similarly, by substituting Eq. (13) into Eqs (7) and (8), the resulting grid values $v_{j}^{n}$ are used in the finite difference schemes as approximation to the continuous solutions $v(x, t)$ to Eqs (7) and (8) respectively. Naturally, Eqs (7) and (8) need condition Eq. (10) only.

\section{Steady-state responses}

For periodical transverse loads, the beam may move periodically after a short transition time. Such periodical motion is referred as the steady-state response. Usually, the periodical steady-state responses are not sensitive to initial conditions. In the following, the beam center displacement is used to represent the beam motion. For an even $N, v_{N / 2}$ is the beam center displacement. The final steady-state response is actually independent the initial conditions, while the various initial condition may leads to different transient processes.

Let $k_{f}=0.8$ and $\gamma=0.5$. In this case, the natural frequency of the linear elastic system can be numerically solved [6] as $\omega_{1}=8.320$. As $k_{1}$ represents the effect of nonlinearity, it is called the nonlinear coefficient. Because, physically, $k_{2}$ is rather small [22], its variation has no discernible effect on the steady-state response. Therefore, in the following calculations, choose $k_{2}=0$ for the sake of simplicity. Based on the numerical solutions of Eqs (6), (7), and (8), the differences between the models can be investigated via the time history near the resonances. Figure 1 illustrates the time history in the first resonances for $b=0.5, \alpha=0.0001, k_{1}=100$. In Fig. 1, the dots, the dash-dot lines and the solid lines stand for the numerical solutions to Eqs (6), (7) and (8). The numerical results demonstrate that the time history of Eq. (7) is different from those of Eqs (6) and (8) for the initial state, and the amplitude of Eq. (7) is smaller than those of Eqs (6) and (8) for the steady state periodic response.

Based on the numerical solutions of Eqs (6), (7), and (8), the differences between the models can be investigated via the amplitude-frequency (described by $\mu=\omega-\omega_{1}$ ) response curves near the resonances. Figure 2 illustrates the amplitude-frequency response curves in the first resonances for different nonlinear coefficients, load amplitudes, and viscosity coefficients. In Fig. 2, the dots, the dash-dot lines and the solid lines stand for the numerical solutions to Eqs (6), (7) and (8). The numerical results demonstrate that three models qualitatively predict the same tendencies with the changing parameters, while quantitatively, there are certain differences and results from Eq. (8) are closer to those from Eq. (6). 


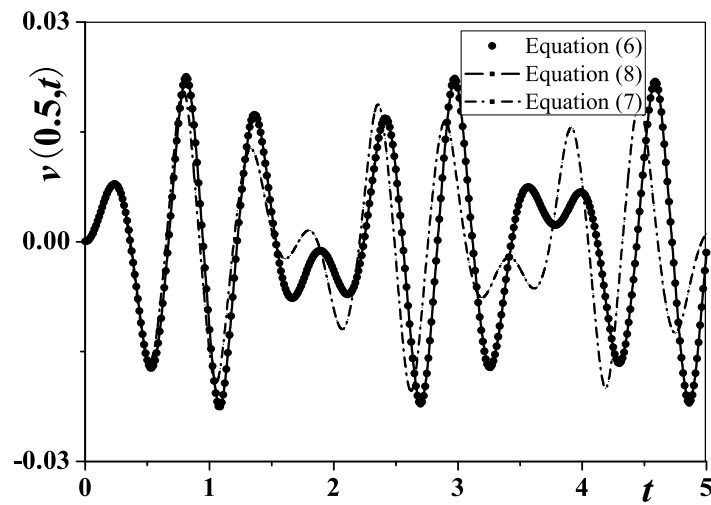

(a) the initial state

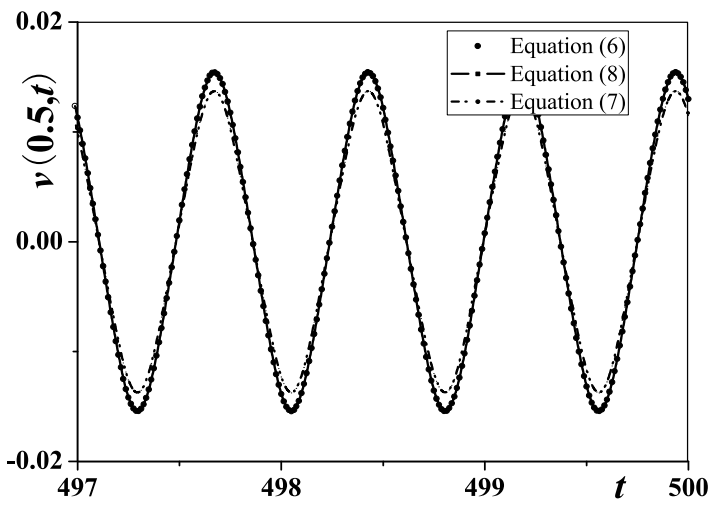

(b) the steady state

Fig. 1. The time history calculated from three models in the first resonance.

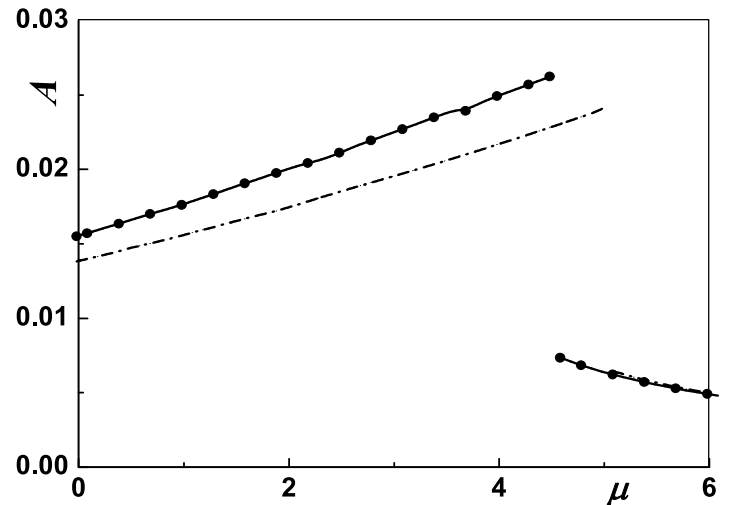

(a) $b=0.5, \alpha=0.0001, k_{1}=100$

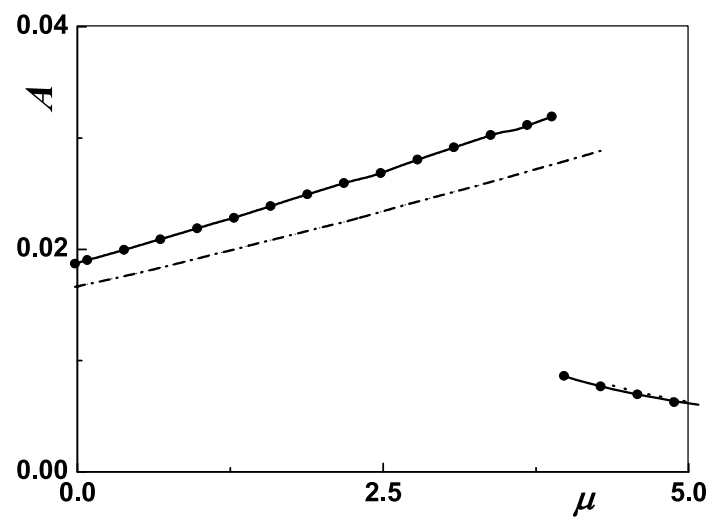

(c) $b=0.5, \alpha=0.0001, k_{1}=75$

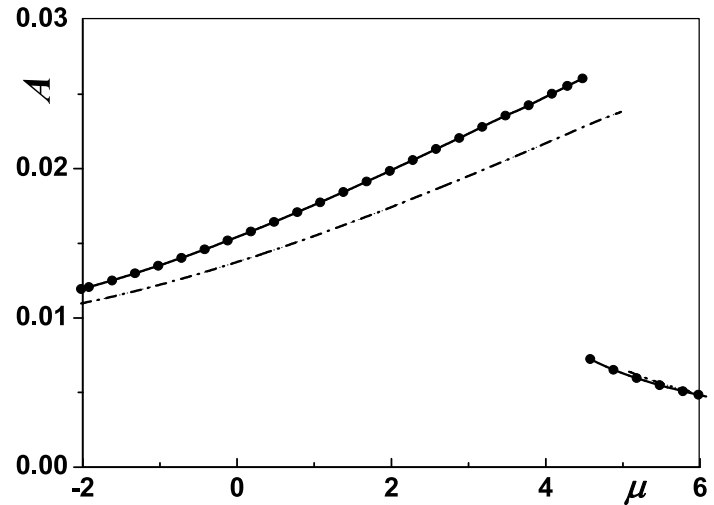

(b) $b=0.5, \alpha=0.0005, k_{1}=100$

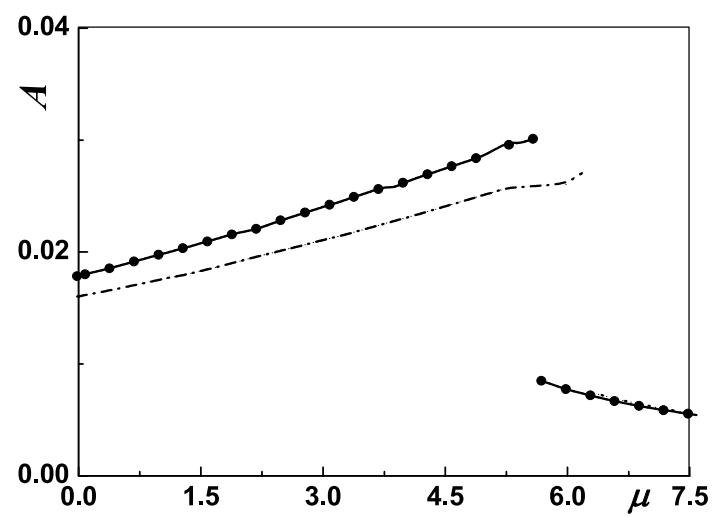

(d) $b=0.75, \alpha=0.0001, k_{1}=100$

Fig. 2. The steady-state responses calculated from three models in the first resonance.

Based on the numerical solutions of Eqs (6), (7), and (8), the differences between the models can be investigated via the shape of beams in the resonances. Figure 3 shows the shapes of beams in the first resonances for different nonlinear coefficients, load amplitudes, and viscosity coefficients. In Fig. 3, the dots, the dash-dot lines and the solid lines stand for the numerical solutions to Eqs (6), (7), and (8), respectively. In all cases, results from Eq. (7) or 


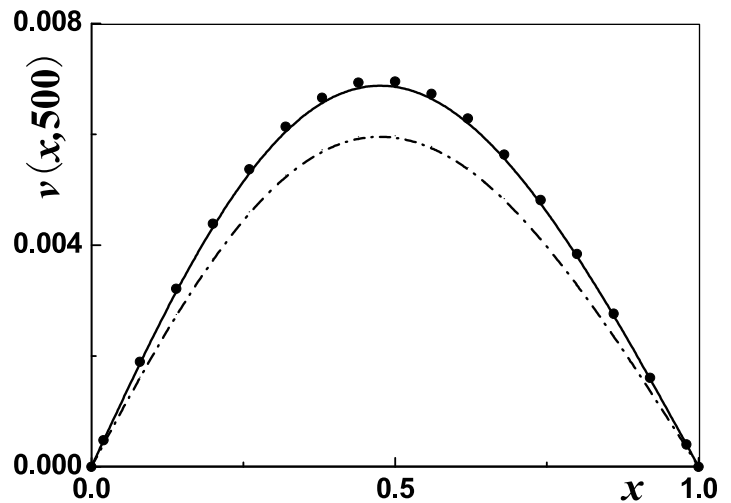

(a) $b=0.5, \alpha=0.0001, k_{1}=100$

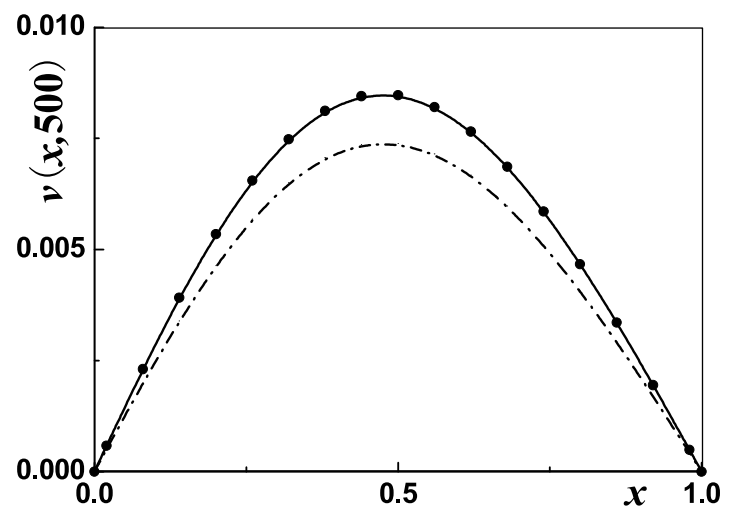

(c) $b=0.5, \alpha=0.0001, k_{1}=0.75$

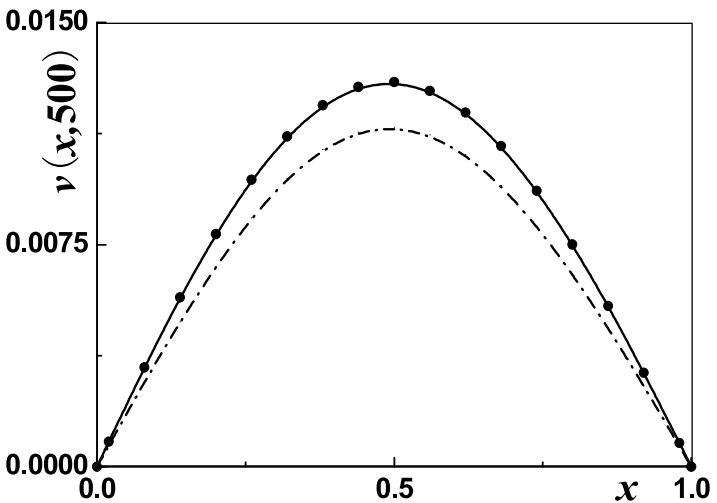

(b) $b=0.5, \alpha=0.0005, k_{1}=100$

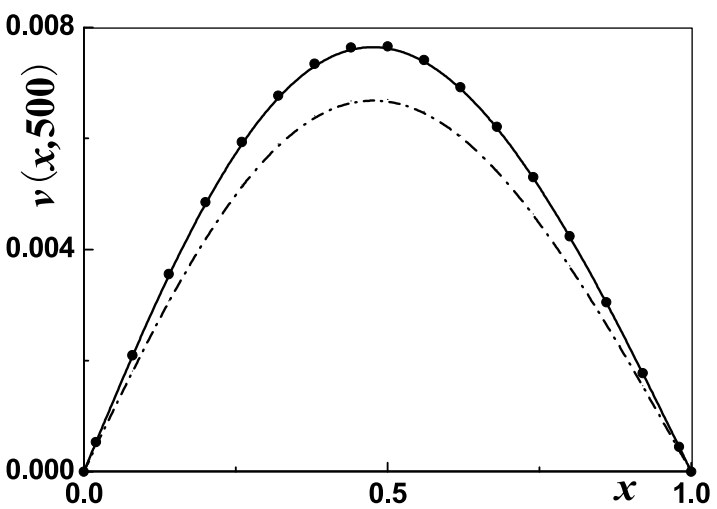

(d) $b=0.75, \alpha=0.0001, k_{1}=100$

Fig. 3. The shape of beam calculated from three models in the first resonance.

Eq. (8) are close to those from Eq. (6), and those from Eq. (8) are closer. In addition, Fig. 3 shows the slight lack of symmetry in the beam deflections, and the fact illustrates the effect of the axial motion.

It should be remarked that, in the above examples, the largest displacement is almost $10 \%$ of the whole beam span. Therefore, even if the vibration is reasonable large, the transverse modes (7) and (8), especially Eq. (8), can still yield satisfactory results.

\section{Conclusions}

This paper is devoted to compare the nonlinear models of transverse vibration of axially moving viscoelastic beams subjected external transverse loads via steady-state periodical response. The planar vibration is governed by a set of coupled nonlinear partial-differential equations. The coupled governing equations can be reduced to two kinds of governing equations of transverse vibration. One is a nonlinear partial-differential equation, and the other is a nonlinear integro-partial-differential equation. The finite difference scheme is developed to solve these governing equations numerically. The steady-state responses can be calculated from the three models. The investigation leads to the following conclusions£"under the periodical transverse loads, there are steady-state periodic responses in transverse vibration. Qualitatively, the three models predict the same tendencies with the changing parameters. Quantitatively, in the view of both the center amplitude and the beam shape, the nonlinear integro-partial-differential equation yields the results closer to those from the coupled equations. The differences among the three models are not sensitive to the nonlinear coefficients, the dynamic viscosity, and the external load amplitudes. 


\section{Acknowledgments}

The work described in this paper was supported by the National Natural Science Foundation of China (No. 10902064), the National Outstanding Young Scientists Fund of China (No. 10725209), Shanghai Subject Chief Scientist Project (No. 09XD1401700), Shanghai Leading Talent Program, Shanghai Leading Academic Discipline Project (No. S30106), and the program for Changjiang scholars and Innovative Research Team in University (No. IRT0844).

\section{References}

[1] A.L. Thurman and C.D. Mote Jr., Free, periodic, nonlinear oscillation of an axially moving strip, Journal of Appled Mechanics 36 (1969), 83-91.

[2] J.A. Wickert, Non-linear vibration of a traveling tensioned beam, International Journal of Non-Linear Mechanics 27 (1992), $503-517$.

[3] G. Suweken and W.T. Van Horssen, On the weakly nonlinear, transversal vibrations of a conveyor belt with a low and time-varying velocity, Nonlinear Dynamics 31 (2003), 197-223.

[4] B. Ravindra and W.D. Zhu, Low dimensional chaotic response of axially accelerating continuum in the supercritical regime, Archive of Applied Mechanics 68 (1998), 195-205.

[5] F. Pellicano, A. Fregolent, A. Bertuzzi and F. Vestroni, Primary and parametric non-linear resonances of a power transmission belt, Journal of Sound and Vibration 244 (2001), 669-684.

[6] L.Q. Chen and X.D. Yang, Steady-state response of axially moving viscoelastic beams with pulsating speed: comparison of two nonlinear models, International Journal of Solid and Structure 42 (2005), 37-50.

[7] L.Q. Chen and H. Ding, Steady-state responses of axially accelerating viscoelastic beams: approximate analysis and numerical confirmation, Science in China G $\mathbf{5 1}$ (2008), 1707-1721.

[8] L.N. Panda and R.C. Karb, Nonlinear dynamics of a pipe conveying pulsating fluid with combination, principal parametric and internal resonances, Journal of Sound and Vibration 309 (2008), 375-406.

[9] R.G. Parker and Y. Lin, Parametric instability of axially moving media subjected to multifrequency tension and speed fluctuations, ASME Journal of Applied Mechanics 68 (2001), 49-57.

[10] F. Pellicano and F. Vestroni, Complex dynamic of high-speed axially moving systems, Journal of Sound and Vibration 258 (2002), $31-44$.

[11] L.Q. Chen and X.D. Yang, Nonlinear free vibration of an axially moving beam: comparison of two models, Journal of Sound and Vibration 299 (2007), 348-354.

[12] H. Ding and L.Q. Chen, Equilibria of axially moving beams in the supercritical regime, Archive of Applied Mechanics (2009) DOI: 10.1007/s00419-009-0394-y.

[13] T.Z. Yang, B. Fang, Y. Chen and Y.X. Zhen, Approximate solutions of axially moving viscoelastic beams subject to multi-frequency excitations, International Journal of Non-Linear Mechanics 44 (2009), 230-238.

[14] M.H. Ghayesh and S.E. Khadem, Rotary inertia and temperature effects on non-nonlinear vibration, steady-state response and stability of an axially moving beam with time-dependent velocity, International Journal of Mechanical Sciences 50 (2008), 389-404.

[15] M.H. Ghayesh and S. Balar, Non-linear parametric vibration and stability analysis for two dynamic models of axially moving Timoshenko beams, Applied Mathematics Modelling (2009), doi: 10.1016/j.apm.2009.12.019

[16] M.H. Ghayesh and S. Balar, Non-linear parametric vibration and stability of axially moving visco-elastic Rayleigh beams, International Journal of Solids and Structures 45 (2008), 6451-6467.

[17] W. Zhang and C.Z. Song, Higher-dimensional periodic and chaotic oscillations for viscoelastic moving belt with multiple internal resonances, International Journal of Bifurcation and Chaos 17 (2007), 1637-1660.

[18] S.H. Chen, J.L. Huang and K.Y. Sze, Multidimensional Lindstedt-Poincaré method for nonlinear vibration of axially moving beams, Journal of Sound and Vibration 306 (2007), 1-11.

[19] K. Marynowski and T. Kapitaniak, Zener internal damping in modelling of axially moving viscoelastic beam with time-dependent tension, International Journal Non-Linear Mechanics 42 (2007), 118-131.

[20] H. Ding and L.Q. Chen, On two transverse nonlinear models of axially moving beams, Science in China E 52 (2009), 743-751.

[21] L.Q. Chen and H. Ding, Two nonlinear models of a transversely vibrating string, Archive of Applied Mechanics 78 (2008), $321-328$.

[22] L.Q. Chen and H. Ding, Steady-state transverse response in coupled planar vibration of axially moving viscoelastic beams, ASME Journal of Vibration and Acoustics 132 (2010), 011009. 

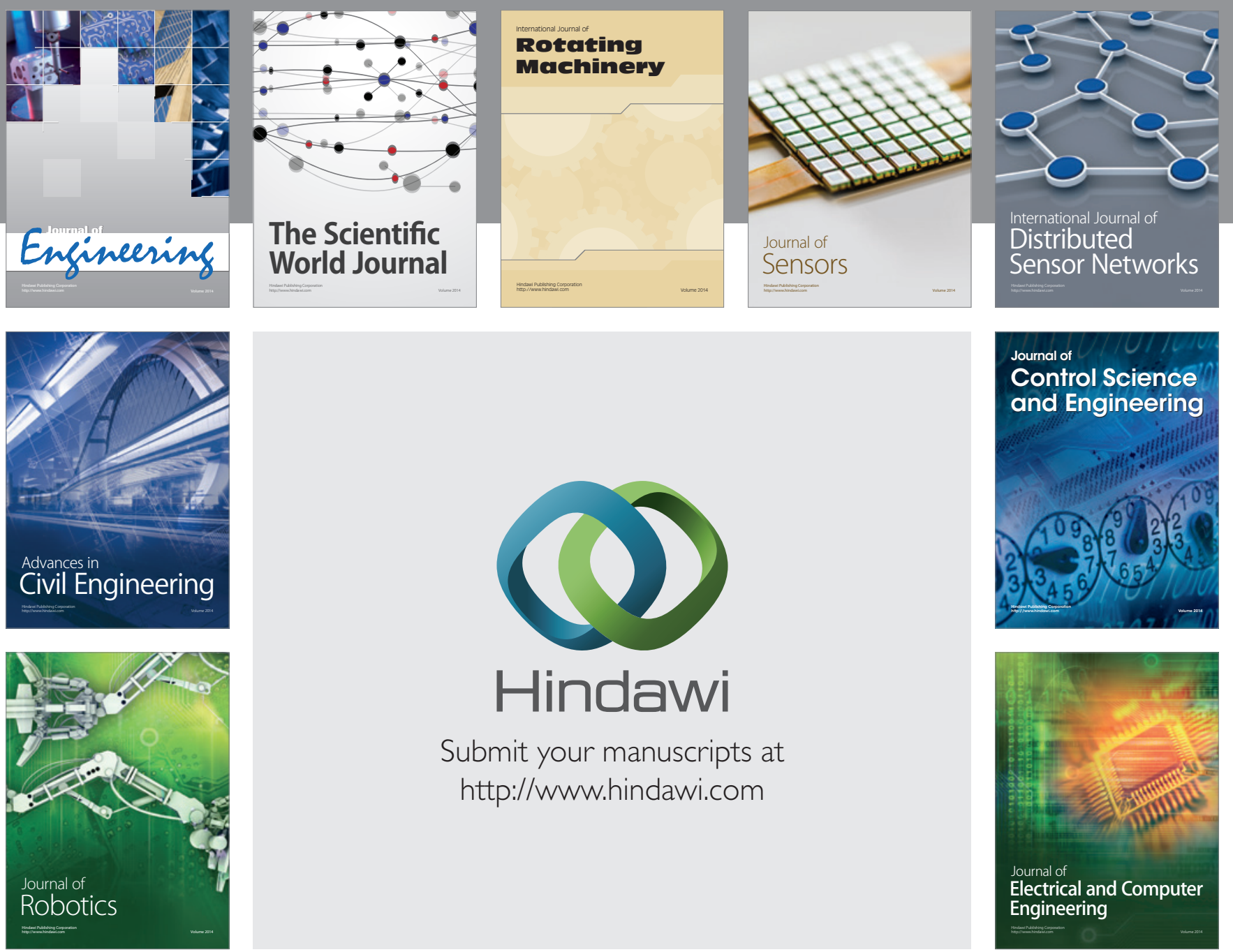

Submit your manuscripts at

http://www.hindawi.com
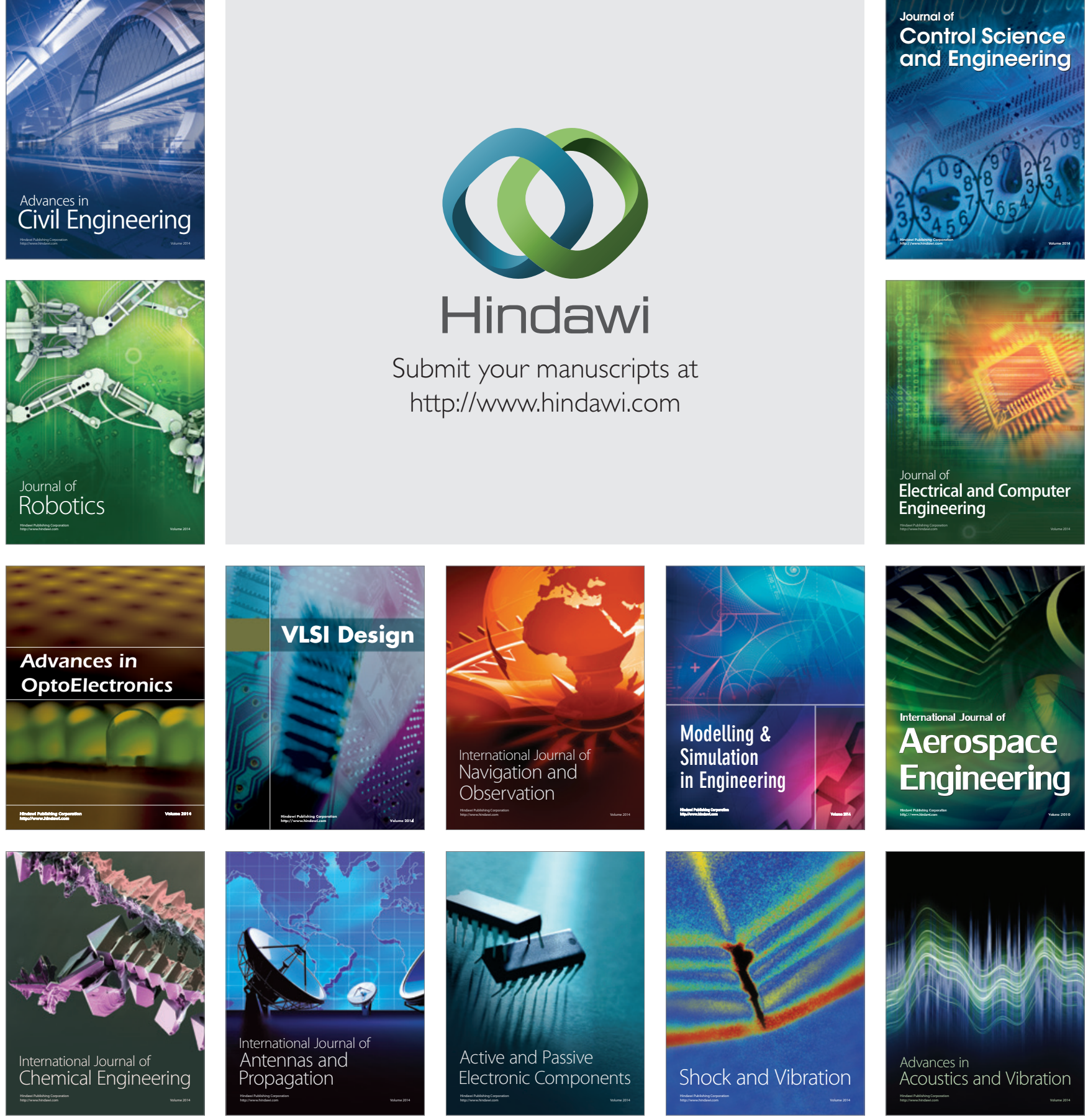